\title{
Cost-effectiveness analysis of dacomitinib versus gefitinib for the first-line therapy of patients with EGFR mutation-positive non-small-cell lung cancer in the United States and China
}

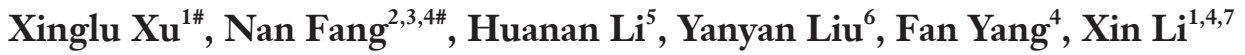 \\ ${ }^{1}$ Department of Clinical Pharmacy, School of Pharmacy, Nanjing Medical University, Nanjing, China; ${ }^{2}$ School of Pharmaceutical Science and \\ Technology, Tianjin University, Tianjin, China; ${ }^{3}$ Center for Social Science Survey and Data, Tianjin University, Tianjin, China; ${ }^{4}$ Department of \\ Health Policy, School of Health Policy and Management, Nanjing Medical University, Nanjing, China; ${ }^{5}$ Department of Pharmacy Practice, Thomas \\ J. Long School of Pharmacy, University of the Pacific, Stockton, California, USA; ${ }^{6}$ Department of Human Resources, the Affiliated Drum Tower \\ Hospital of Nanjing University Medical School, Nanjing, China; ${ }^{7}$ Center for Global Health, School of Public Health, Nanjing Medical University, \\ Nanjing, China \\ Contributions: (I) Conception and design: X Li, N Fang; (II) Administrative support: X Li, Y Liu; (III) Provision of study materials or patients: X Xu, \\ N Fang; (IV) Collection and assembly of data: X Xu, N Fang, F Yang, X Li; (V) Data analysis and interpretation: X Li, X Xu, H Li, N Fang; (VI) \\ Manuscript writing: All authors; (VII) Final approval of manuscript: All authors. \\ \#These authors contributed equally to this work. \\ Correspondence to: Xin Li, PhD; Fan Yang, PhD. Nanjing Medical University, 101 Longmian Avenue, Jiangning District, Nanjing 211166, China. \\ Email: xinli@njmu.edu.cn; yangfan512@njmu.edu.cn.
}

Background: This study aimed to investigate the cost-effectiveness of dacomitinib and gefitinib for the firstline treatment of advanced non-small-cell lung cancer (NSCLC) in epidermal growth factor receptor (EGFR) mutation-positive patients from the perspective of healthcare systems in the United States and China.

Methods: A Markov model, which included 3 health states over 10 years, was established in this study. The state transition probabilities and clinical data were extracted from the ARCHER 1050 trial (dacomitinib versus gefitinib in patients with EGFR mutation-positive advanced NSCLC). Health utilities were derived from published literature. Based on the healthcare system payer's perspective in the United States and China, the cost data were estimated from local pricing or the relevant literature. The health outcomes are expressed by quality-adjusted life years (QALYs). All costs and incremental cost-effectiveness ratios (ICERs) are presented in US dollars. One-way sensitivity analysis and probabilistic sensitivity analysis were performed to test the robustness of the results.

Results: In the United States, compared with gefitinib, dacomitinib yielded an additional 0.55 QALYs, while the ICERs were $\$ 600.69$ per QALY. The cost of dacomitinib was the most influential parameter. The willingness payment curve showed that dacomitinib was cost-effective at the $\$ 100,000 / \mathrm{QALY}$ willingnessto-pay (WTP) threshold. Meanwhile, when the WTP threshold was higher than \$200,000/QALY, the probability of dacomitinib being the best treatment plan was more than $80 \%$. In China, compared with gefitinib, dacomitinib was associated with a mean healthcare savings of $\$ 160,173.27$ and 0.41 additional QALYs per patient, which was a dominant intervention over a 10-year time horizon. The cost of progressive disease was shown to have the strongest impact on the results. Dacomitinib had more than a $90 \%$ probability of being chosen as the preferred therapy when the Chinese WTP threshold was \$27,000/QALY.

Conclusions: As the first-line treatment for EGFR mutation-positive NSCLC, dacomitinib is likely to be more cost-effective than gefitinib from the healthcare system's perspective in the United States and China.

Keywords: Non-small-cell lung cancer (NSCLC); cost-effectiveness; dacomitinib; EGFR mutation-positive

Submitted Oct 19, 2020. Accepted for publication Feb 28, 2021.

doi: 10.21037/atm-20-6992

View this article at: http://dx.doi.org/10.21037/atm-20-6992 


\section{Introduction}

Lung cancer is currently among those malignant tumors with the highest morbidity and mortality rate (1). According to 2018 statistics, lung cancer incidence is one of the highest in both China and the United States, thus imposing a heavy burden on patients and society (2). Moreover, most lung cancer patients are already in advanced stages at the time of diagnosis and constitute nearly $25 \%$ of all cancer deaths (3). Non-small-cell lung cancer (NSCLC) accounts for about $85 \%$ of lung cancer patients, whereas approximately $10-15 \%$ of Caucasian patients and above $40 \%$ of Asian patients have epidermal growth factor receptor (EGFR) mutations, which are highly responsive to EGFR-tyrosine kinase inhibitors (EGFR-TKIs) (4). Therefore, the targeted therapy of EGFR-TKIs has emerged as the first-line standard treatment option for such patients. Currently, the National Comprehensive Cancer Network (NCCN) recommends first-generation EGFR-TKIs such as gefitinib, erlotinib, and afatinib as the first-line treatment options for patients with advanced EGFR-mutated NSCLC (5).

Gefitinib is a first-generation EGFR-TKI and has been widely used in the first-line treatment of NSCLC patients for over 10 years. Research has shown that in comparison to chemotherapy with pemetrexed plus carboplatin, gefitinib can significantly prolong progression-free survival (PFS) [11.9 months, $95 \%$ confidence interval (CI), 9.1-14.6 vs. 5.7 months, 95\% CI, 5.2-6.3] (6). Clinical trials have also shown that although gefitinib is associated with more grade $3 / 4$ liver dysfunction than afatinib and erlotinib; its overall incidence of adverse events (AEs) is lower, suggesting it has good safety $(7,8)$.

Dacomitinib is a new type of oral irreversible secondgeneration EGFR-TKI. Wu et al. (9) conducted an international randomized phase III trial (ARCHER 1050) in 2017, which compared the safety and efficacy of dacomitinib with gefitinib in the first-line treatment of advanced NSCLC patients with the EGFR mutation. Notably, the trial gathered 452 eligible patients from 7 countries or regions and randomly divided them into two groups (1:1) to receive oral dacomitinib $45 \mathrm{mg} /$ day $(\mathrm{n}=227)$ or oral gefitinib $250 \mathrm{mg} /$ day $(\mathrm{n}=225)$ both in 28 -day cycles until disease progression or until the time patients dropped out. According to the findings, dacomitinib significantly prolonged PFS as compared to gefitinib. Based on this trial, dacomitinib (produced by Pfizer Inc.) was approved for marketing by the US Food and Drug Administration (FDA) as a new EGFR-TKI in the United States in September 2018. About 8 months later, in May 2019, it was launched as a first-line treatment for EGFR mutation-positive adults with advanced NSCLC in China.

Although anticancer medicines are in great demand, they are expensive and often beyond the patient's financial means to acquire, especially the new targeted agents. As dacomitinib has only been on the market for a short time, only a few studies worldwide have been conducted on its cost-effectiveness. The amendment to the National Basic Medical Insurance List has recently emphasized using health technology assessment (HTA) evidence in China. The underlying value of medicine for cancers can be measured by health gains and costs, which can provide the government or the insurer an idea of the appropriate price for obtaining medicine to achieve value for money (10). However, considering the country-specific nature of the cost variables, the economic evaluation findings' generalizability and transferability are limited (11). This study investigated the cost-effectiveness of dacomitinib and gefitinib for the first-line treatment of advanced EGFRmutation-positive NSCLC patients in the United States and China. The United States and China are high- and middle-income countries, which could improve the generalizability and transferability of the economic outcomes. The economic assessment findings may provide an evidence base for the comparative value of dacomitinib and gefitinib. We present the following article in accordance with the CHEERS reporting checklist (available online http://dx.doi. org/10.21037/atm-20-6992).

\section{Methods}

\section{Overview}

The decision tree and Markov models were used to estimate the clinical costs and outcomes of dacomitinib or gefitinib first-line treatment for EGFRmutation-positive NSCLC patients in the United States and China. The 3 states of the Markov model include progression-free survival (PFS), progressive disease (PD), and death (Figure 1). We assumed that all patients were in PFS in the initial stage of the model and would be able to stay in PFS or transition to the PD or death stage. However, those who moved to the PD stage could only stay in PD or progress to the death stage. The Markov cycle length was 28 days, consistent with the treatment schedule in the ARCHER 1050 trial (9). We set up a total of 130 cycles of simulation, which was the equivalent of 10 years. In each Markov cycle, the model redistribution of hypothetical patients among the 3 health states was based on the transition probabilities.

Quality-adjusted life-years (QALYs) and direct medical 


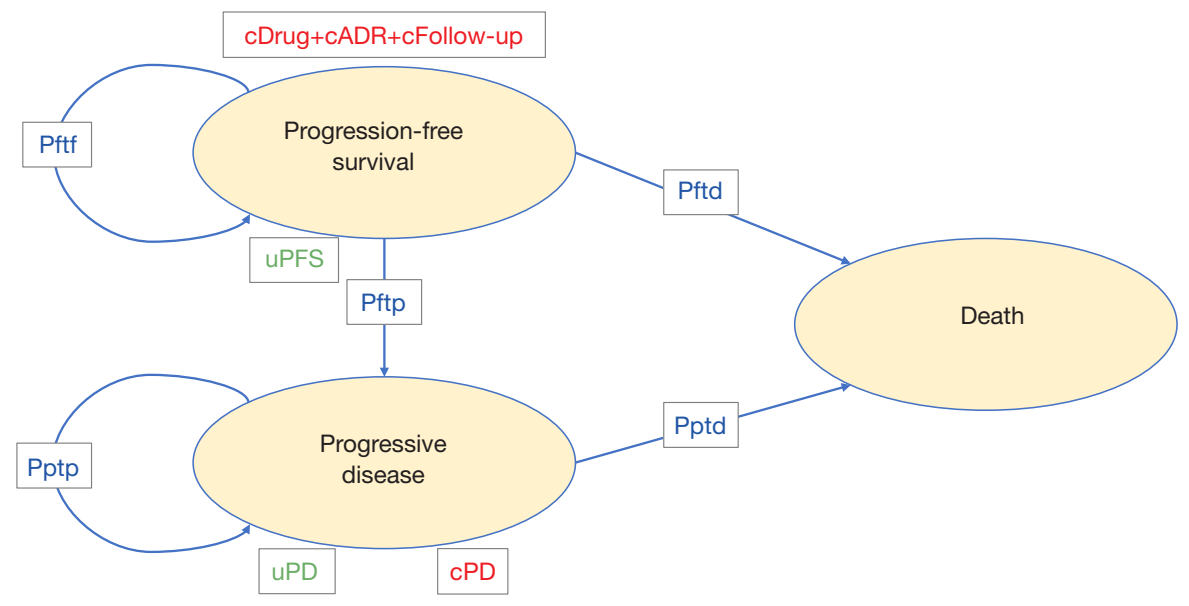

Figure 1 Diagrammatic representation of the Markov Model. Pftf, probability from PFS to PFS; Pftd, probability from PFS to death; Pftp, probability from PFS to PD; Pptp, probability from PD to PD; Pptd, probability from PD to death; uPFS, utility of PFS; uPD, utility of $\mathrm{PD}$; cDrug, cost of drugs; cADR, cost of ADR relative rescue therapy; cFollow-up, cost of follow-up therapy; cPD, cost of PD therapy.

costs were examined, and incremental cost-effectiveness ratio (ICER) was calculated to evaluate the outcomes. When the ICER value is less than the threshold, the treatment is considered affordable and economical. The formula of ICER is as follows:

$$
\text { ICER }=\frac{\text { Cost (dacomitinib) }- \text { Cost (gefitinib) }}{\text { QALYs (dacomitinib) }- \text { QALYs (gefitinib) }}
$$

The costs are expressed in US dollars, and the exchange rate of Chinese yuan to USD in 2019 was used (1 CNY=0.1420 USD). All costs and QALYs had an annual discount of $3 \%$ and $5 \%$ in the United States and China, respectively (12). We used $\$ 100,000$ as the American willingness-to-pay (WTP) threshold and 3-times China's per capita gross domestic product (GDP) in 2018 $(\$ 27,519.60)$ as the Chinese WTP threshold, according to previous research literature $(13,14)$. Furthermore, to test the results' robustness, one-way sensitivity analysis and probabilistic sensitivity analysis were performed.

The study was conducted in accordance with the Declaration of Helsinki (as revised in 2013). This study used mathematical modeling and was not an active clinical trial; therefore, no approval was required from the Institutional Research Ethics Board.

\section{Statistical analysis}

\section{Clinical data}

The patient data were collected from the ARCHER 1050 trial (9), which included a $75 \%$ Asian population and had certain significance for this study. The basic demographic characteristics of the trial are well-balanced between the two groups. Based on the results of phase III clinical trials, the median PFS of dacomitinib and gefitinib was 14.7 months (95\% CI: 11.1-16.6) and 9.2 months (95\% CI: 9.1-11.0), respectively [hazard ratio (HR): 0.59 (0.47-0.74); $\mathrm{P}<0.0001$ ], whereas the median overall survival (OS) was 34.1 and 26.8 months, respectively [HR: 0.760 (0.582-0.993); $\mathrm{P}=0.044$ ] (15). Regarding the adverse drug events (AEs), diarrhea, rash, paronychia, and abnormal alanine transaminase (ALT) were examined in the study. Grade 3 or higher severe AEs (SAEs) were included because their impact on health utility and quality of life has been demonstrated. The probabilities of AEs were also obtained from the ARCHER 1050 trial. Moreover, the incidence of AEs in the clinical trial was converted into an incidence per cycle measure. Finally, the average differences in efficacy endpoints (PFS, $\mathrm{PD}$, and $\mathrm{OS}$ ) between dacomitinib and gefitinib were examined using the area under the curve (AUC) (16). The $t$-test was performed on the data of average PFS and OS in the clinical trial.

\section{Transition probabilities}

The Weibull model was used to fit the transition probabilities of PFS to PD and of PD to death. by using $\mathrm{R}$ for Statistical Computing version 3.4.4 ( $\mathrm{R}$ version for Statistical Computing, Vienna, Austria). PFS and OS curves were fit using GetData Graph Digitizer software (Version 
Table 1 Scale $(\lambda)$ and Shape $(\gamma)$ parameters of PFS and OS curves

\begin{tabular}{lcc}
\hline Parameters & Value & Reference \\
\hline PFS curve & $\lambda=0.031826 ; \gamma=1.057874$ & The Weibull model \\
Dacomitinib & $\lambda=0.014916 ; \gamma=1.517880$ & The Weibull model \\
Gefitinib & $\lambda=0.004085 ; \gamma=1.430855$ & The Weibull model \\
OS curve & $\lambda=0.007903 ; \gamma=1.238186$ & The Weibull model \\
Dacomitinib & & 0.000563364 \\
Gefitinib & 0.000568438 & (16) \\
Age-adjusted death rate & & (17) \\
The United States & & \\
China & &
\end{tabular}

PFS, progression-free survival; OS, overall survival.

2.26, getdata-graph-digitizer.com). Age-adjusted death rates in the United States and China (per 100,000 standard population) were used to calculate the transition probability of PFS to death $(17,18)$. The formula of transition probability calculation was as follows:

$$
P(t)=1-\exp \left[\lambda(t-1)^{\gamma}-\lambda t^{\gamma}\right]
$$

where $\lambda$ is the scale of the distribution, $\gamma$ is the shape of the distribution, and $\mathrm{t}$ is the Markov cycle.

All the parameters and values of transition probabilities are presented in Table 1 .

Due to the lack of patient-level data in the ARCHER 1050 trial, we could not calculate the non-cancer-specific cumulative incidence of death. Therefore, the competing risks were not considered in this study.

\section{Medical costs}

Only direct medical costs were calculated to estimate the treatment costs, and included the drug's costs, the management of AEs, the PD therapy, and follow-up. The cost data were estimated from the perspectives of the healthcare systems in the United States and China. The recommended dosages of dacomitinib and gefitinib were derived from the ARCHER 1050 trial and the drug instructions $(9,19,20)$. Dacomitinib and gefitinib were orally administered at doses of 45 and $250 \mathrm{mg}$, respectively, once a day until disease progression $(19,20)$. Therefore, the cost of subsequent therapies was calculated based on published studies. By genetic testing, NSCLC patients who progressed after first-line treatment were divided into 3 subgroups, which were de novo Thr790Met (T790M)-positive, anaplastic lymphoma kinase (ALK)/c-Met proto-oncogene(c-Met)positive, and no mutations. The overall positive T790M and ALK/c-Met rates were $52.2 \%$ and $20.49 \%$, respectively (21). Patients who were T790M-positive were given osimertinib mesylate tablets ( $80 \mathrm{mg} /$ day orally), ALK/c-Met-positive patients were given crizotinib tablets $(500 \mathrm{mg} /$ day orally), and patients without mutations were given chemotherapy (paclitaxel and cisplatin, 21 days/cycle) according to the guidelines and expert recommendations (22). The following formula calculated the total cost of progression disease: $52.20 \% \times$ cost of T790M-positive therapy per cycle + $20.49 \% \times$ cost of ALK/c-Met-positive therapy per cycle + $(1-52.20 \%-20.49 \%) \times$ cost of chemotherapy per cycle.

Follow-up costs included routine blood examination and biochemical testing every cycle, and tumor marker test and computed tomography every 2 cycles. Patient assistance programs were not considered in this study. The costs of each treatment's SAE's were calculated by multiplying the incidence of SAEs by the costs of managing the SAE per event. PD therapy included gene examination and drugs. Costs calculation was the same as that of SAEs.

Data on the costs of PFS, SAEs, PD therapy, and the follow-up in China were collected from local hospitals by conducting a questionnaire survey of local clinical experts. The prices of dacomitinib and gefitinib were also obtained from local hospitals. Meanwhile, follow-up prices (routine blood examination, biochemical testing, tumor marker testing, and computed tomography) and PD therapy were also acquired from local centers and the published literature.

The prices of dacomitinib and gefitinib in the United 
Table 2 Costs, utilities, and discount rates of the USA and China in the model

\begin{tabular}{|c|c|c|c|c|c|c|}
\hline Parameter & \multicolumn{3}{|c|}{ The United States } & \multicolumn{3}{|c|}{ China } \\
\hline \multicolumn{7}{|c|}{ Costs per cycle (US \$) } \\
\hline Dacomitinib & $7,385(5,539-9,231)$ & Gamma & Red Book & $1,731\left(865^{\star}-2,163\right)$ & Gamma & Local charge \\
\hline Gefitinib & $6,218(4,664-7,773)$ & Gamma & Red Book & 163 [122-204] & Gamma & (27) \\
\hline ADR of Gef. & $0.35(0.26-0.44)$ & Gamma & $(9,28-31)$, OCC & 3.38 (2.54-4.23) & Gamma & (9), local charge \\
\hline Follow-up & 437 [328-546] & Gamma & (32) & 269 [201-336] & Gamma & Local charge \\
\hline PD therapy & $12,636(9,477-15,795)$ & Gamma & (33) & $28,644(21,483-35,805)$ & Gamma & $(34-36)$ \\
\hline \multicolumn{7}{|l|}{ Utility } \\
\hline \multicolumn{7}{|c|}{ Discount rate per year (\%) } \\
\hline Cost & $3(0-6)$ & - & (12) & $5(0-8)$ & - & $(12)$ \\
\hline Outcome & $3(0-6)$ & - & (12) & $5(0-8)$ & - & (12) \\
\hline
\end{tabular}

*, in China, due to the price negotiation between China's National Healthcare Security Administration (NHSA) and pharmaceutical companies, the dacomitinib range was set to $50-125 \%$. OCC, outpatient care center; ADR, adverse drug reaction; PD, progressive disease; PFS, progression-free survival; OS, overall survival.

States were collected from Red Book Online 2019 (Truven Health Analytics, New York, NY, USA) (23).

The costs of SAEs, PD therapy, and follow-up in the United States were collected from the outpatient healthcare costs in 2015 based on the American Outpatient Care Center (OCC) and public literature (24).

\section{Health utility}

Health utility was used to calculate QALYs of patients, which were obtained from the report by Nafees et al., who used the time-trade off technique to determine the utilities of NSCLC $(25,26)$. PFS and PD utility in the United States was 0.84 and 0.166 , respectively, and those in China were 0.804 and 0.321 , respectively $(12,25,26)$. However, the impact of SAEs on QALY was not explicitly included in the model because it was assumed that treatment-specific utility values had captured the burden of toxicity. All the base case costs and utilities are shown in Table 2.

\section{Sensitivity analysis}

One-way sensitivity analysis and probabilistic sensitivity analysis (PSA) were performed to test the model's uncertainty and robustness. In the sensitivity analysis, 10 parameters were examined to form the tornado diagrams. Due to China's National Healthcare Security Administration (NHSA) and pharmaceutical companies' strategic price negotiations, dacomitinib's price was discounted by $50 \%$ in China (27). Other parameters were discounted between $15 \%$ to $25 \%$ (Table 2). Next, 1,000 Monte-Carlo simulations were performed in the probabilistic analysis, after which a cost-effectiveness plane was drawn to show the distribution of random variables. The cost-effectiveness curves represented the patients' acceptability at different WTP thresholds in both countries.

Both Markov model and sensitivity analysis were developed in Microsoft Excel (Microsoft Corporation, Redmond, WA).

\section{Ethical statement}

The study was conducted in accordance with the Declaration of Helsinki (as revised in 2013). This study used mathematical modeling and was not an active clinical trial; therefore, no approval was required from the Institutional Research Ethics Board. 
Table 3 Average PFS, PD, and OS of the clinical data and the model

\begin{tabular}{|c|c|c|c|c|c|c|c|c|c|}
\hline Items & \multicolumn{2}{|c|}{ Average PFS (m) } & $\begin{array}{c}\text { Mean } \\
\text { difference }\end{array}$ & \multicolumn{2}{|c|}{ Average OS (m) } & $\begin{array}{c}\text { Mean } \\
\text { difference }\end{array}$ & \multicolumn{2}{|c|}{ Average PD (m) } & $\begin{array}{c}\text { Mean } \\
\text { difference }\end{array}$ \\
\hline Clinical data* (48 months) & 18.33 & 13.12 & 5.21 & 30.29 & 29.32 & 0.97 & 11.96 & 16.20 & -4.24 \\
\hline Model outcomes (120 months) & 24.81 & 13.89 & 10.92 & 41.52 & 44.47 & -2.95 & 16.71 & 30.58 & -13.87 \\
\hline
\end{tabular}

*, a significant difference $(\mathrm{P}<0.05)$ was found in average PFS between dacomitinib and gefitinib in the clinical trial, but no statistical difference was found in average OS between dacomitinib and gefitinib in the clinical trial $(\mathrm{P}>0.05)$. PFS, progression-free survival; OS, overall survival; $\mathrm{PD}$, progressive disease.

\section{Results}

The average PFS, PD, and OS of the clinical data and the Weibull model outcomes are presented in Table 3. A significant difference $(\mathrm{P}<0.05)$ was found in average PFS between dacomitinib and gefitinib in the clinical trial, but no statistical difference was found in average OS between dacomitinib and gefitinib in the clinical trial $(\mathrm{P}>0.05)$.

As shown in Table 3, the mean PFS over the 120-month horizon was 24.81 months for dacomitinib and 13.89 months for gefitinib, with a mean difference of 10.92 months. The mean OS over the 120-month horizon was 41.52 months for dacomitinib and 44.47 months for gefitinib, with a mean difference of 2.95 months. The mean PD over the 120 -month horizon was 16.71 months for dacomitinib and 30.58 months for gefitinib, with a mean difference of 13.87 months.

Fitted curves were compared with the Kaplan-Meier (K-M) survival curves in ARCHER 1050 trial, as seen in Figures 2 and 3. These figures also show the reconstructed $\mathrm{K}-\mathrm{M}$ curves along with fitted Weibull models for OS and PFS. As the figures show, the reconstructed curves fit well with the clinical trial's K-M curves, which demonstrated that the Weibull models accurately validated the OS and PFS data.

\section{Base case analysis}

As shown in Table 4, compared with gefitinib, the NSCLC treatment strategy with dacomitinib resulted in an increased QALY of 0.55 (1.89 vs. 1.34 QALY) per patient in the 10-year cumulative cycle, with an additional cost of $\$ 330.14$ (\$557,894.97 vs. $\$ 557,564.83)$ in the United States. Compared to gefitinib, the ICER of dacomitinib was $\$ 600.69$ per QALY. The value of ICER was much lower than the WTP threshold $(\$ 100,000)$, which showed that dacomitinib was cost-effective in the United States. Dacomitinib saved $\$ 160,173.27$ but provided 0.41 more
QALYs in China, which indicated that it was more costeffective than gefitinib as the first-line treatment for EGFR-mutated NSCLC in China based on the cited WTP threshold of $\$ 27519.60$.

\section{Sensitivity analysis}

The results of the deterministic sensitivity analyses are shown in Figures 4 and 5. The tornado diagrams were made to aggregate the results of sensitive variables from high to low. In the top 5 influencing parameters of ICERs in the United States and China, 4 parameters were found to be similar. They were (I) the cost of dacomitinib; (II) the cost of PD therapy; (III) the utility of PFS; and (IV) the cost discount rate per year. However, there were some differences in the ranks of influence on the economic outcomes between both countries. The one-way sensitivity analysis results showed that the most sensitive variable was dacomitinib's cost in the United States. On the other hand, PD therapy cost was observed to have the most obvious impact on the ICERs in China. Other variables, such as the utility of PFS, cost discount rate per circle, and cost of follow-up, had a moderate or mild impact on the ICER results.

As illustrated in Figure 4, even if the price of dacomitinib or gefitinib in the United States increased by $25 \%$, its ICER value would still be less than a $\$ 100,000 \mathrm{WTP}$ threshold. Therefore, dacomitinib was stably cost-effective in the United States. As the value of ICER was negative, the costeffectiveness of dacomitinib in China was certain.

PSA was also conducted to test the robustness of all the input parameters used in this study. The distribution of utility value and transition probability was set as the model's beta distribution, whereas the distribution of cost was set as the gamma distribution. Then, 1,000 simulations were executed by setting different distributions for the parameters. Scatter plots and acceptable cost-effectiveness 


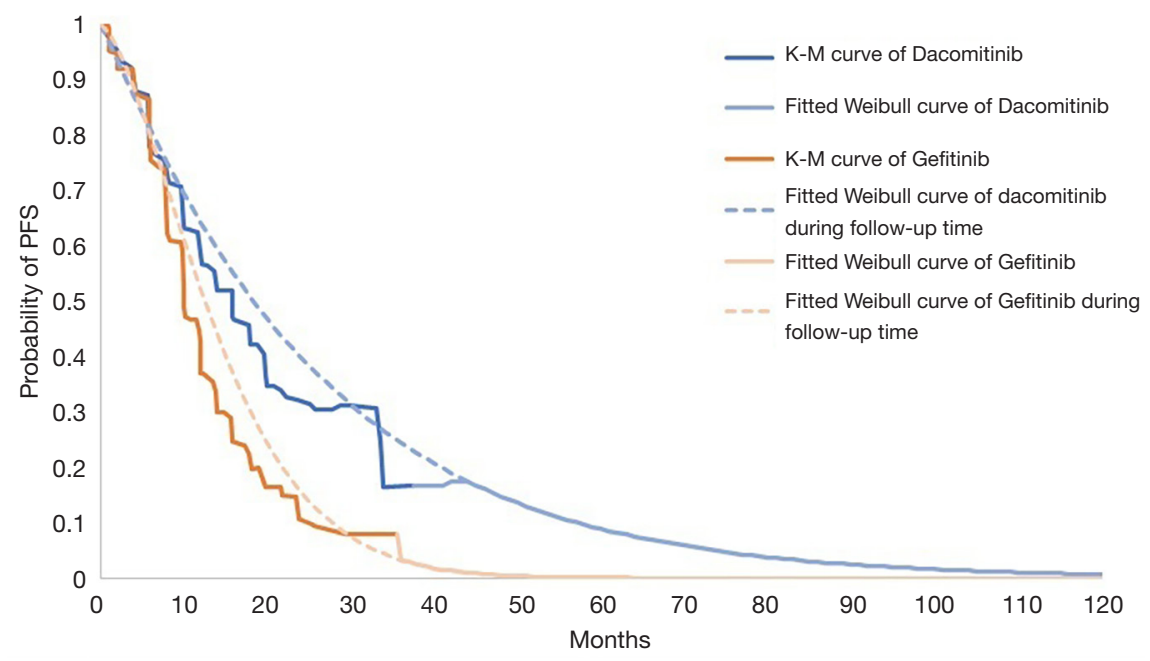

Figure 2 Reconstructed K-M curves and fitted Weibull curves for PFS of dacomitinib and gefitinib. PFS, progression-free survival.

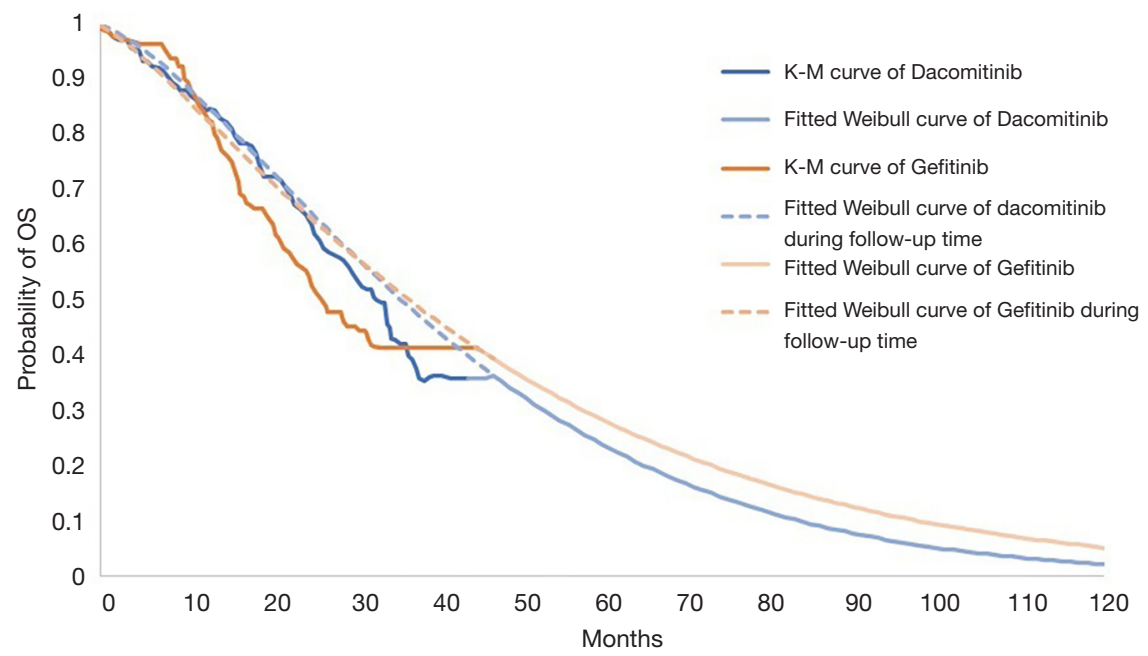

Figure 3 Reconstructed K-M curves and fitted Weibull curves for OS of dacomitinib and gefitinib. OS, overall survival.

Table 4 Base case results of dacomitinib versus gefitinib in the USA and China

\begin{tabular}{lccccccc}
\hline \multirow{2}{*}{ Group } & \multicolumn{3}{c}{ The United States } & & \multicolumn{2}{c}{ China } \\
\cline { 2 - 4 } \cline { 6 - 8 } & Costs $(\$)$ & QALY & ICER & Costs(\$) & QALY & ICER \\
\hline Dacomitinib & $557,894.97$ & 1.89 & $\$ 600.69$ per QALY & $834,869.94$ & 2.08 & - \\
Gefitinib & $557,564.83$ & 1.34 & & $995,043.21$ & 1.67 & \\
\hline
\end{tabular}




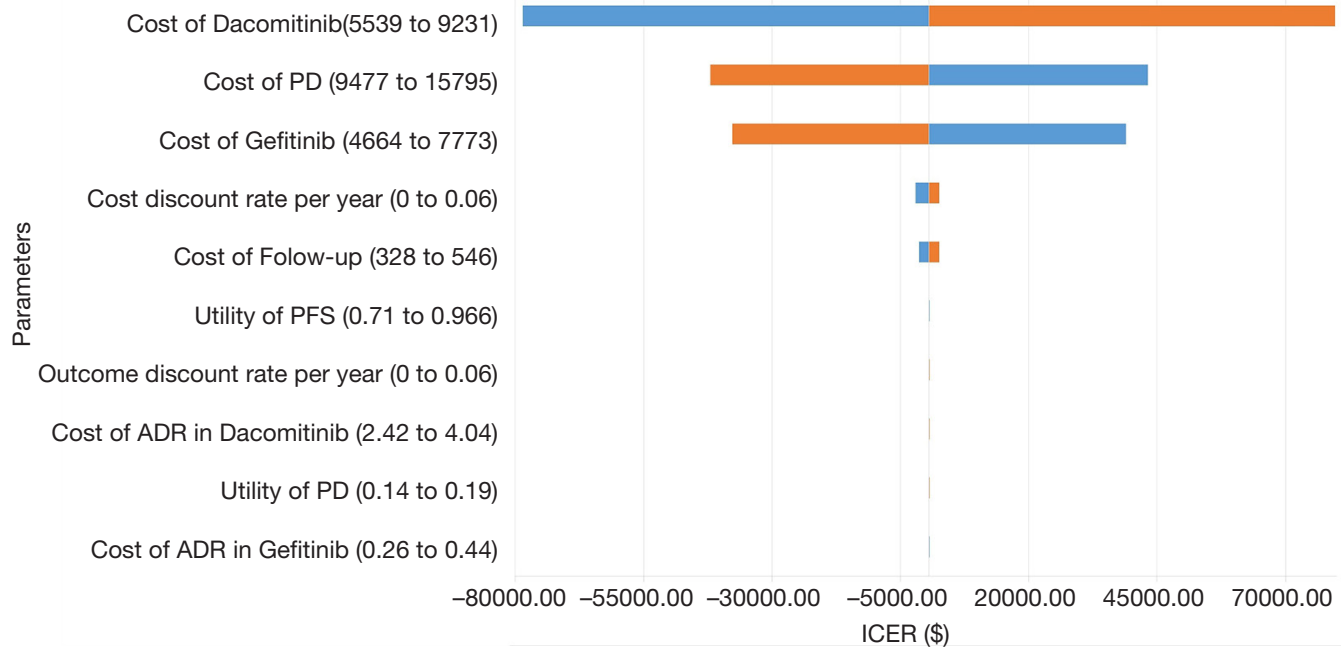

Figure 4 One-way sensitivity analysis of the United States.

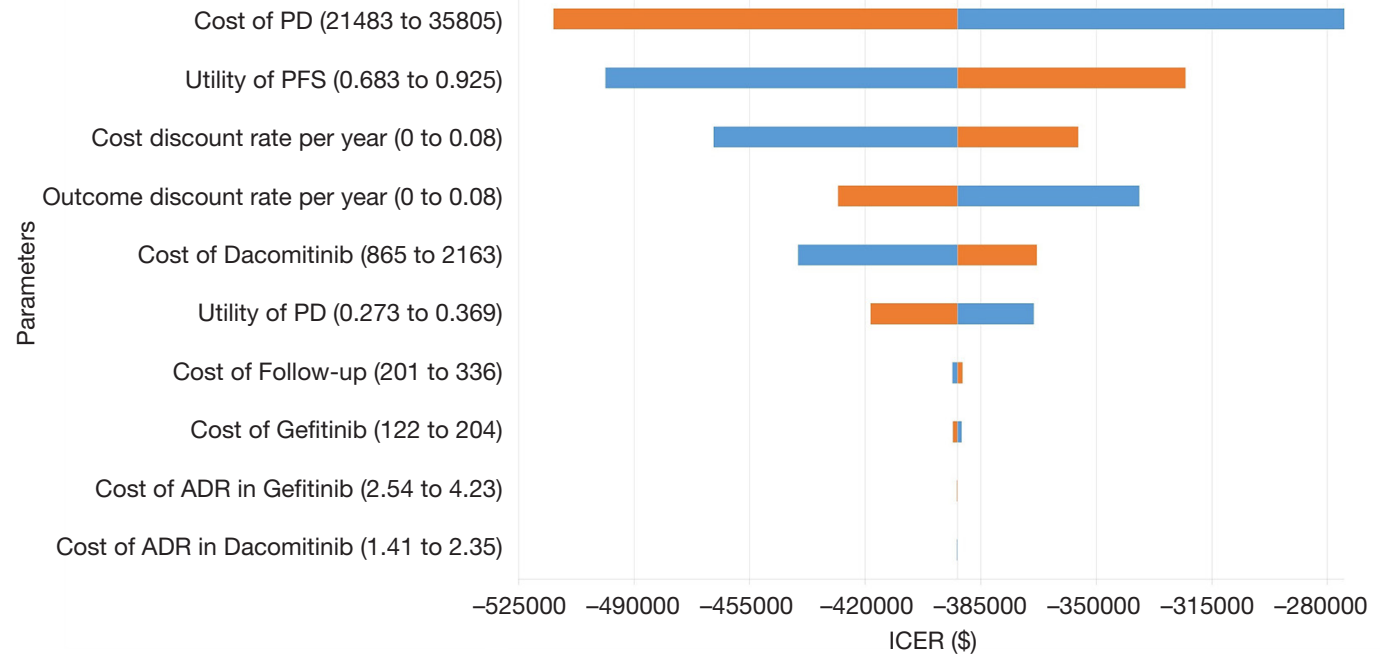

Figure 5 One-way sensitivity analysis of China.

curves were drawn based on these simulations (Figures 6,7). As illustrated in Figures 6 and 7, the figures' diagonal lines represent the two countries' WTP threshold lines. Scatter points below the slash were considered cost-effective and acceptable. Figure 6 shows that when the WTP in the US was $\$ 100,000$, about two-thirds of the points were below the threshold. The acceptability curve (Figures 8,9) also indicates that regardless of how the threshold changed in the United States, dacomitinib's acceptability was always higher than that of gefitinib. Also, as the WTP increased, its acceptability gradually increased. In China, fewer than
$10 \%$ of the scatter points are over the threshold line, indicating more than an $80 \%$ probability of dacomitinib being dominant over gefitinib.

\section{Discussion}

In recent years, progress in novel oncology drugs has increased the healthcare costs in high- and medium-income countries, including the United States and China (37). In this situation, there is growing concern that the launch of innovative anticancer medicines will impose a substantial 


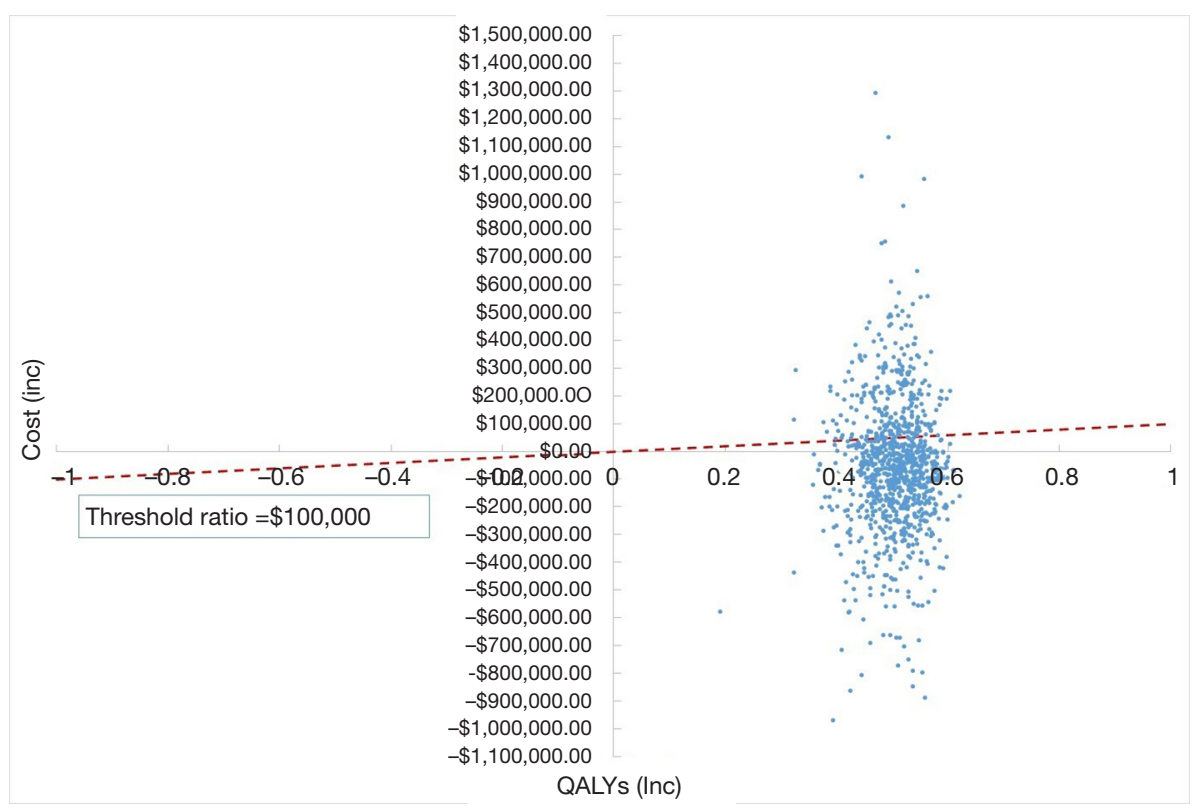

Figure 6 Scatter plots and cost-effectiveness plane of the United States.



Figure 7 Scatter plots and cost-effectiveness plane of China.

burden on the national healthcare budget (37). In an era of rapidly developing novel pharmaceuticals and limited drug resources, establishing the value of novel anticancer drugs is imperative for optimizing resource allocation and improving the accessibility of innovative anticancer medicines (38).

Although dacomitinib therapy's clinical efficacy has been discovered in a clinical trial, there are some concerns that dacomitinib's price does not reflect its expected clinical benefits in the United States and China. In this study, a Markov model was adapted to assess dacomitinib's costeffectiveness against gefitinib as the first-line treatment of advanced NSCLC. Due to the short time to launch the 


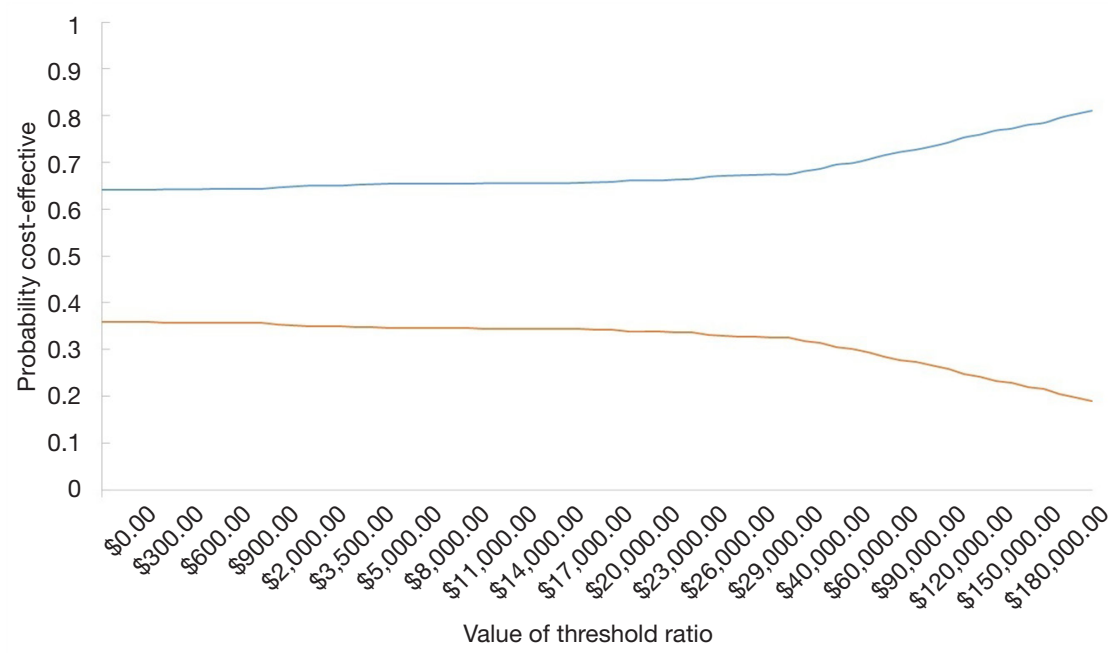

Figure 8 Acceptability curves of dacomitinib and gefitinib in the United States. Blue line: acceptance curve of dacomitinib; Orange line: acceptance curve of gefitinib.

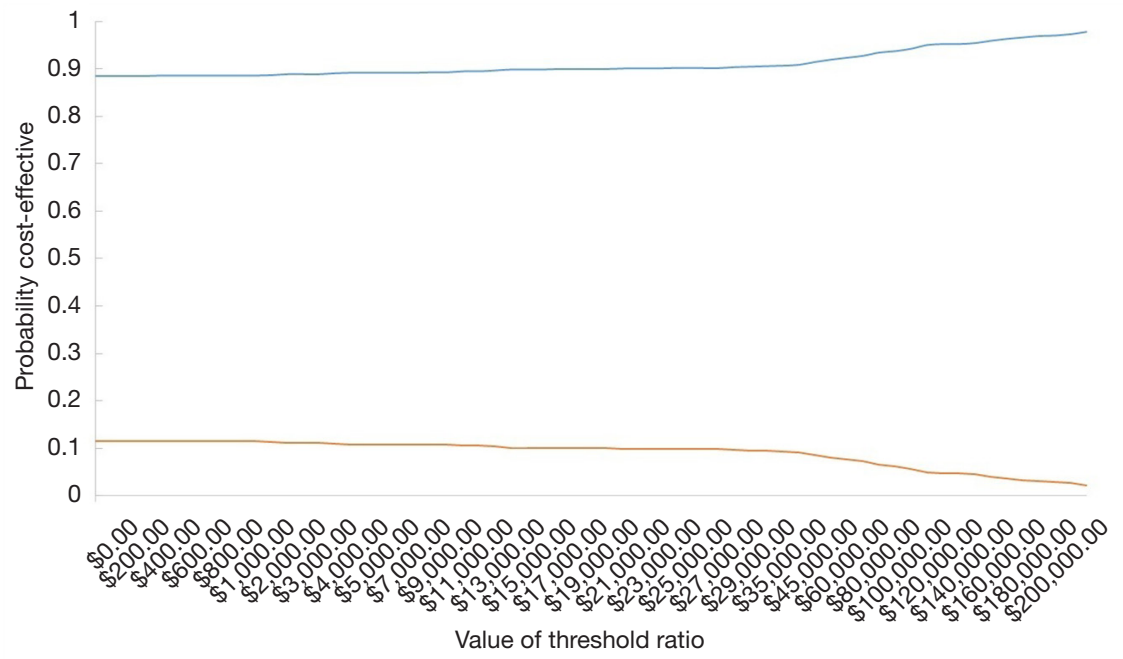

Figure 9 Acceptability curves of dacomitinib and gefitinib in China. Blue line: acceptance curve of dacomitinib; Orange line: acceptance curve of gefitinib.

novel anticancer medicine in the United States and China, few studies have focused on dacomitinib's economic aspects. This economic evaluation focused on dacomitinib as the first-line treatment for patients with EGFR mutations from a healthcare system perspective in both countries. It may have substantial reference value for the health sector's decision-making in high- and middle-income settings such as the United States and China to expand healthcare access at a reasonable cost. The ARCHER 1050 study, an international, head-to-head, multicenter, randomized phase III clinical trial, demonstrated that dacomitinib was more clinically efficacious than gefitinib. However, due to its higher price and greater frequency of SAEs, clinicians, and patients may have concerns about dacomitinib therapy. The results of this study showed that although dacomitinib had a longer PFS (14.7 vs. 9.2 months) and OS (34.1 vs. 26.8 months) than gefitinib (9), the QALY obtained was also much longer than that of gefitinib.

Meanwhile, the total cost of dacomitinib was much higher than gefitinib in the United States $(\$ 557,894.97$ 
vs. $\$ 557,564.83)$ due to the higher cost of research and development as well as for treatments of SAEs. Compared with gefitinib treatment, the dacomitinib therapy produced an average incremental cost-effectiveness ratio of $\$ 600.69$ / QALY, which was smaller than the threshold value of $\$ 100,000$ per QALY in the United States. On the other hand, the total cost of dacomitinib treatment was cheaper than that of gefitinib (\$834,869.94 vs. $\$ 995,043.21)$ in China, but the final QALY was higher than that of gefitinib. Therefore, the dacomitinib treatment proved to be a costeffective therapeutic strategy in both the United States and China, a finding which may help inform the healthcare systems of high- and medium-income countries. The acceptability curve also showed that most certainties were achieved at a WTP threshold of \$100,000/QALY in the United States and \$27,519.60/QALY in China.

In the United States context, the results of deterministic one-way sensitivity analysis showed that the cost of dacomitinib treatment had the greatest influence on the model outcome. An economic evaluation study that investigated the cost-effectiveness of osimertinib to treat advanced NSCLC with an EGFR T790M mutation after the failure of first-line EGFR TKI therapy in the United States also found that the cost of novel anticancer drug exhibited the highest impact on the ICER for osimertinib versus chemotherapy (12). A similar study also found that novel target anticancer drugs' price had an obvious impact on ICERs (39). This could be attributable to the higher acquisition costs of dacomitinib in the United States. For instance, the current local price of dacomitinib in the United States is nearly 4 times that in China, suggesting that there is potential to reduce dacomitinib's price. It might be the most effective approach for reducing the ICERs below the WTP threshold to achieve more favorable economic outcomes in the United States. In China's context, the results of deterministic one-way sensitivity analysis showed that the most sensitive variable was the cost of PD treatment. The finding can be reasonably explained. Dacomitinib was found to significantly improve PFS over gefitinib in the first-line treatment of patients with EGFR mutation-positive NSCLC, which meant that the dacomitinib treatment could reduce the duration of the PD state. The cost of PD treatment for advanced NSCLC patients in China was substantial, thus increasing the barriers to affordability. Therefore, reducing the PD state duration could be a suitable approach for providing a cost-effective alternative to gefitinib. Notably, the PD state's treatment paradigms included the use of more expensive novel drugs such as osimertinib and crizotinib. Thus, it is necessary to promote centralized strategic price negotiations with pharmaceutical companies in the Chinese healthcare system.

Other researchers have performed similar studies on EGFR-TKIs, including on osimertinib, afatinib, and erlotinib. For instance, Chouaid et al. (40) conducted a cost-effectiveness analysis of afatinib versus gefitinib in France. The study showed that the first-line afatinib gained an ICER of $€ 45,211$ per QALY compared with gefitinib (0.170 QALY of an incremental cost of $€ 7,697)$, whose probability of being cost-effective for NSCLC patients with EGFR mutation was $100 \%$. Gu et al. (41) reached a similar conclusion that afatinib could confer additional $0.382,0.216$, and 0.174 QALYs with an extra $\$ 7,930, \$ 3,680$, and $\$ 2,818$ in costs in comparison to pemetrexed plus cisplatin (PC), gefitinib, and erlotinib, yielding ICERs of \$20,758, \$17,693, and $\$ 16,197$ per QALY, respectively.

The study had some limitations that are worth discussing. (I) Modeling with Weibull functions to fit the PFS and OS curves beyond the trial's follow-up duration is an inevitable limitation. Also, the Weibull model includes competing risks between lung cancer-specific and non-cancer-specific incidence of death. Due to competing risks, the transition probabilities of PD to death could have been exaggerated. However, due to the model's appropriate suitability and probabilistic analysis, the model's certainty was guaranteed to some extent. Most importantly, the competing risks could be virtually eliminated by the ICER method. (II) Furthermore, drug discounts and patient assistance programs were not considered in this study, making the costs slightly higher than those in the real-world in the long term. (III) The negative effects of ADR on utility were excluded in the calculation. Nevertheless, based on oneway sensitivity analysis results, we can speculate that the negative effects of ADR on utility had a negligible impact on the base-case analysis.

\section{Conclusions}

This study used a Markov model to evaluate the economic outcomes of 2 first-line treatment options for patients with advanced EGFR mutation-positive NSCLC. It was found that, from the perspective of the healthcare systems of the United States and China, first-line dacomitinib treatment is likely to be considered cost-effective for patients with advanced EGFR mutation-positive NSCLC. Also, the results of one-way sensitivity analysis and probability 
sensitivity analysis were found to be relatively stable. If the cost of gefitinib or PD therapy were to be reduced, then gefitinib may also become a viable option in the United States and China.

\section{Acknowledgments}

We are grateful for J. Gray and J. Chapnick from the AME Editing Service for their English language editing work.

Funding: This study was supported grants from the National Natural Science Foundation of China (No. 72074123 and 71673147), the China Medical Board (No. 17-277), and the Joint Project between Southeast University and Nanjing Medical University (No. 2018DN0023). The funding agencies had no role in the study design, data collection, analysis, decision to publish, or manuscript preparation.

\section{Footnote}

Reporting Checklist: The authors have completed the CHEERS reporting checklist. Available online http:// dx.doi.org/10.21037/atm-20-6992

Data Sharing Statement: Available online http://dx.doi. org/10.21037/atm-20-6992

Conflicts of Interest: All authors have completed the ICMJE uniform disclosure form (available online http://dx.doi. org/10.21037/atm-20-6992). The authors have no conflicts of interest to declare.

Ethical Statement: The authors are accountable for all aspects of the work in ensuring that questions related to the accuracy or integrity of any part of the work are appropriately investigated and resolved. The study was conducted in accordance with the Declaration of Helsinki (as revised in 2013). This study used mathematical modeling and was not an active clinical trial; therefore, no approval was required from the Institutional Research Ethics Board.

Open Access Statement: This is an Open Access article distributed in accordance with the Creative Commons Attribution-NonCommercial-NoDerivs 4.0 International License (CC BY-NC-ND 4.0), which permits the noncommercial replication and distribution of the article with the strict proviso that no changes or edits are made and the original work is properly cited (including links to both the formal publication through the relevant DOI and the license).
See: https://creativecommons.org/licenses/by-nc-nd/4.0/.

\section{References}

1. Chen $\mathrm{W}$, Zheng R, Baade PD, et al. Cancer statistics in China, 2015. CA Cancer J Clin 2016,66:115-32.

2. Feng RM, Zong YN, Cao SM, et al. Current cancer situation in China: good or bad news from the 2018 Global Cancer Statistics? Cancer Commun (Lond) 2019;39:22.

3. American Cancer Society. About non-small cell lung cancer. 2016. Available online: https://www.cancer.org/ content/dam/CRC/PDF/Public/8703.00.pdf. Accessed April 14, 2018.

4. Shi Y, Au JS, Thongprasert S, et al. A prospective, molecular epidemiology study of EGFR mutations in Asian patients with advanced non-small-cell lung cancer of adenocarcinoma histology (PIONEER), J Thorac Oncol 2014;9:154-62.

5. NCCN Practice Guidelines in Oncology. Non-small cell lung cancer, version 4.2019. Available online: nccn.org.

6. Han B, Jin B, Chu T, et al. Combination of chemotherapy and gefitinib as first-line treatment for patients with advanced lung adenocarcinoma and sensitive EGFR mutations: A randomized controlled trial. Int J Cancer 2017;141:1249-56.

7. Park K, Tan EH, O’Byrne K, et al. Afatinib versus gefitinib as first-line treatment of patients with EGFR mutationpositive non-small-cell lung cancer (LUX-Lung 7): a phase 2B, openlabel, randomised controlled trial. Lancet Oncol 2016;17:577-89.

8. Soria JC, Felip E, Cobo M, et al. Afatinib versus erlotinib as second-line treatment of patients with advanced squamous cell carcinoma of the lung (LUX-Lung 8): an open-label randomised controlled phase 3 trial. Lancet Oncol 2015;16:897-907.

9. Wu YL, Cheng Y, Zhou X, et al. Dacomitinib versus gefitinib as first-line treatment for patients with EGFRmutation-positive non-small-cell lung cancer (ARCHER 1050): a randomised, open-label, phase 3 trial. Lancet Oncol 2017;18:1454-66.

10. Si L, Xu L, Chen M, et al. Using strategic price negotiations to contain costs and expand access to medicines in China. BMJ Glob Health 2020;5:e02256.

11. Petrou P. A systematic review of economic evaluations of tyrosine kinase inhibitors of vascular endothelial growth factor receptors, mammalian target of rapamycin inhibitors and programmed death- 1 inhibitors in metastatic renal 
cell cancer. Expert Rev Pharmacoecon Outcomes Res 2018;18:255-65.

12. Wu B, Gu X, Zhang Q. Cost-effectiveness of osimertinib for EGFR mutation-positive non-small cell lung cancer after progression following first-line EGFR TKI therapy. J Thorac Oncol 2018;13:184-93.

13. Neumann PJ, Cohen JT, Weinstein MC. Updating costeffectiveness--the curious resilience of the \$50,000-perQALY threshold. N Engl J Med 2014;371:796-7.

14. Lu S, Ye M, Ding L, et al. Cost-effectiveness of gefitinib, icotinib, and pemetrexed-based chemotherapy as firstline treatments for advanced non-small cell lung cancer in China. Oncotarget 2017;8:9996-10006.

15. Mok TS, Cheng Y, Zhou X, et al. Improvement in Overall Survival in a Randomized Study That Compared Dacomitinib With Gefitinib in Patients With Advanced Non-Small-Cell Lung Cancer and EGFR-Activating Mutations. J Clin Oncol 2018;36:2244-50.

16. Chris B. The Mean, Median, and Confidence Intervals of the Kaplan-Meier Survival Estimate-Computations and Applications. Am Stat 2009;63:78-80.

17. Age-adjusted death rates in the U.S. in 2017 (per 100,000 standard population). Available online: https://www.cdc. gov/nchs/data/databriefs/db328_tables-508.pdf\#2

18. National Bureau of Statistics of China: National Economy was Generally Stable in 2019 with Main Projected Targets for Development Achieved. Available online: http://www.stats.gov.cn/english/PressRelease/202001/ t20200117_1723398.html

19. Iressa (gefitinib) for oral use. AstraZeneca. July 2015. Available online: https://www.azpicentral.com/iressa/iressa. pdf\#page=1. Accessed April 14, 2018.

20. Drug instruction for Vizimpro (dacomitinib). Pfizer. May 2019. Available online: Pfizer.com.cn

21. Li C, Liu H, Zhang B, et al. Whole-exome sequencing identifies key mutated genes in T790M wildtype/cMETunamplified lung adenocarcinoma with acquired resistance to first-generation EGFR tyrosine kinase inhibitors. J Cancer Res Clin Oncol 2018;144:1079-86.

22. Chinese Guidelines for the Diagnosis and Treatment of Non-Small Cell Lung Cancer with Sensitive Mutations in Epidermal Growth Factor Receptor and Anaplastic Lymphoma Kinase Fusion Genes (Version 2014). Chin J Oncol 2014,36:555-7.

23. Micromedex Solutions. Available online: http://www. micromedexsolutions.com/, accessed Dec 30, 2019.

24. Outpatient CareCenter. Available online: https:// outpatientcc.com/
25. Nafees B, Lloyd AJ, Dewilde S, et al. Health state utilities in non-small cell lung cancer: an international study. Asia Pac J Clin Oncol 2017;13:e195-e203.

26. Nafees B, Stafford M, Gavriel S, et al. Health state utilities for non-small cell lung cancer. Health Qual Life Outcomes 2008;6:84.

27. Notice on the announcement of the results of the centralized procurement of pharmaceuticals in the alliance region. Available online: http://www.smpaa.cn/ gjsdcg/2019/09/30/9040.shtml

28. Elting LS, Shih YCT. The economic burden of supportive care of cancer patients. Support Care Cancer 2004;12:219-26.

29. Carlotto A, Hogsett VL, Maiorini EM, et al. The economic burden of toxicities associated with cancer treatment: review of the literature and analysis of nausea and vomiting, diarrhoea, oral mucositis and fatigue. Pharmacoeconomics 2013;31:753-66.

30. Hirsh V. Managing treatment-related adverse events associated with EGFR tyrosine kinase inhibitors in advanced non-small-cell lung cancer. Curr Oncol 2011;18:126.

31. Sequist LV, JC-H Yang, Yamamoto N, et al. Phase III study of afatinib or cisplatin plus pemetrexed in patients with metastatic lung adenocarcinoma with EGFR mutations. J Clin Oncol 2013;31:3327-34.

32. Klein R, Wielage R, Muehlenbein C, et al. Costeffectiveness of pemetrexed as first-line maintenance therapy for advanced nonsquamous non-small cell lung cancer. J Thorac Oncol 2010;5:1263-72.

33. Fox KM, Brooks JM, Kim J. Metastatic non-small cell lung cancer: costs associated with disease progression. Am J Manag Care 2008;14:565-71.

34. Mok TS, Wu YL, Ahn MJ, et al. Osimertinib or PlatinumPemetrexed in EGFR T790M-Positive Lung Cancer. N Engl J Med 2017;376:629-40.

35. Solomon BJ, Cappuzzo F, Felip E, et al. Intracranial Efficacy of Crizotinib Versus Chemotherapy in Patients With Advanced ALK-Positive Non-Small-Cell Lung Cancer: Results From PROFILE 1014. J Clin Oncol 2016;34:2858-65.

36. Giaccone G, Splinter TA, Debruyne C, et al. Randomized study of paclitaxel-cisplatin versus cisplatin-teniposide in patients with advanced non-small-cell lung cancer. The European Organization for Research and Treatment of Cancer Lung Cancer Cooperative Group. J Clin Oncol 1998;16:2133-41.

37. Saluja R, Arciero VS, Cheng S, et al. Examining Trends in 
Cost and Clinical Benefit of Novel Anticancer Drugs Over Time. J Oncol Pract 2018;14:JOP.17.00058.

38. Teramae F, Yamaguchi N, Makino T, et al. Holistic costeffectiveness analysis of anticancer drug regimens in Japan. Drug Discov Today 2020;25:269-73.

39. Cai H, Zhang L, Li N, et al. Cost-effectiveness of Osimertinib as First-line Treatment and Sequential Therapy for EGFR Mutation-positive Non-small Cell Lung Cancer in China. Clin Ther 2019;41:280-90.

40. Chouaid C, Luciani L, LeLay K, et al. Cost-

Cite this article as: $\mathrm{Xu}$ X, Fang N, Li H, Liu Y, Yang F, Li X. Cost-effectiveness analysis of dacomitinib versus gefitinib for the first-line therapy of patients with EGFR mutation-positive non-small-cell lung cancer in the United States and China. Ann Transl Med 2021;9(9):760. doi: 10.21037/atm-20-6992
Effectiveness Analysis of Afatinib versus Gefitinib for First-Line Treatment of Advanced EGFR-Mutated Advanced Non-Small Cell Lung Cancers. J Thorac Oncol 2017;12:1496-502.

41. Gu X, Zhang Q, Chu YB, et al. Cost-effectiveness of afatinib, gefitinib, erlotinib and pemetrexed-based chemotherapy as first-line treatments for advanced non-small cell lung cancer in China. Lung Cancer 2019;127:84-9. 